\title{
Role of microRNA-15a-5p/TNFAIP3-interacting protein 2 axis in acute lung injury induced by traumatic hemorrhagic shock
}

\author{
FENG ZHOU, ZHIZHEN LIU, HUAZHONG CAI, ZHENJUN MIAO, FAXING WEI and CHAO SONG \\ Department of Emergency, Affiliated Hospital of Jiangsu University, Zhenjiang, Jiangsu 212001, P.R. China
}

Received July 19, 2018; Accepted June 17, 2020

DOI: $10.3892 /$ etm.2020.9130

\begin{abstract}
The present study aimed to investigate the role of microRNA (miR)-15a-5p in the pathogenesis of acute lung injury induced by traumatic hemorrhagic shock (THS), and to explore the underlying molecular mechanism. The expression level of miR-15a-5p was detected using reverse transcription-quantitative (RT-qPCR) and the association between miR-15a-5p and TNFAIP3-interacting protein 2 (TNIP2) was revealed using TargetScan and dual luciferase reporter assays. To investigate the effect of miR-15a-5p on THS-induced acute lung injury, a THS rat model was established. Lung capillary permeability and lung edema were then determined. Moreover, proinflammatory factors in the bronchoalveolar lavage fluid (BALF) and serum of the THS rat model were detected using ELISA. In addition, protein levels in the current study were measured via western blotting. It was revealed that miR-15a-5p was significantly upregulated in both patients with THS and samples from the THS rat model. TNIP2 represents a direct target of miR-15a-5p, and it was downregulated in both patients with THS and the THS rat model. Further analyses indicated that downregulation of miR-15a-5p significantly relieved acute lung injury induced by THS, evidenced by a decreased ratio of Evan's blue dye (EBD) in the BALF to EBD in plasma of THS rats, decreased lung permeability index and reduced lung wet/dry ratio. Inhibition of miR-15a-5p also decreased THS-induced upregulation of pro-inflammatory factors. Furthermore, the data revealed that THS-induced NF- $\kappa \mathrm{B}$ activation in the lung tissues of rats was inhibited by miR-15a-5p knockdown. Moreover, it was demonstrated that all the effects of miR-15a-5p on THS rats were ablated following TNIP2 silencing. Taken together, the data of the current study indicate that miR-15a-5p downregulation serves a protective role in THS-induced acute lung injury via directly targeting TNIP2.
\end{abstract}

Correspondence to: Dr Huazhong Cai, Department of Emergency, Affiliated Hospital of Jiangsu University, 438 Jiefang Road, Zhenjiang, Jiangsu 212001, P.R. China

E-mail: caihz20180719@163.com

Key words: lung injury, trauma-hemorrhagic shock, microRNA15a-5p, TNIP2, inflammatory response

\section{Introduction}

Traumatic hemorrhagic shock (THS) has a high mortality rate of up to $40 \%(1,2)$. The pathophysiological process of THS is complex, involving systemic inflammatory responses and pathological changes, such as microcirculatory disorders, hypovolemia, hypoxemia and oxidative stress (3). The most common complications of THS include systemic inflammatory response syndrome, sepsis, multiple organ dysfunction syndrome and acute lung injury (ALI), which are the primary causes of high mortality $(4,5)$. ALI is characterized by pulmonary edema and micropulmonary nodules, caused by diffuse alveolar capillary injury and resulting in acute, progressive hypoxic respiratory failure (6). Currently, the characteristics of ALI are believed to be the result of extensive destruction of pulmonary vascular endothelial cells and alveolar epithelial cells due to excessive inflammation in the body (7). Patients with ALI exhibit significant morbidity and mortality, poor treatment outcomes and the disease represents a risk factor for clinically critical illness at all ages (8). A key factor in THS-induced injury is the inflammatory response $(9,10)$. Therefore, the majority of studies have focused on the regulation of pro-inflammatory mediators $(11,12)$, and anti-inflammatory agents are a treatment for lung injury induced by THS.

MicroRNAs (miRNAs), a class of small ( 22 nucleotides), non-coding, single-stranded and highly-conserved RNAs, negatively regulate the expression of target genes during various cellular events, including proliferation, apoptosis and differentiation, via binding the 3'UTR of target genes (13-16). MiRNAs have been identified to be involved in various disease types, including cancer, and cardiovascular, nervous system and metabolic diseases, as well as numerous diseases caused by trauma (16-21). In addition, the role of miRNAs in lung injury has been extensively studied (22). However, the role of miRNAs in THS-induced acute lung injury is poorly studied and further research is needed.

MiR-15a-5p has not previously been well characterized. Wang et al (23) reported that miR-15a-5p inhibits endometrial cancer cell growth via regulating the $\mathrm{Wnt} / \beta$-catenin signaling pathway. Chen et al (24) reported that miR-15a-5p regulates cell survival and metastasis of chronic myeloid leukemia by targeting CXCL10. Long et al (25) demonstrated that miR-15a-5p prevents cell proliferation and division in human hepatocellular carcinoma via targeting BDNF. 
Moreover, miR-15a-5p has been revealed as a prognostic biomarker for recurrent colorectal adenocarcinoma (26). In the present study, bioinformatics software analysis revealed that TNFAIP3-interacting protein 2 (TNIP2) was a potential target of miR-15a-5p. The TNIP2 gene encodes a protein identified as a suppressor of NF- $\kappa$ B activation (27). TNIP 2 has been revealed to serve important roles in myocardial injury induced by acute pancreatitis via regulating the inflammatory response (28). It is generally believed that TNIP2 serves an important regulatory role in the $\mathrm{NF}-\kappa \mathrm{B}$ signaling pathway $(28,29)$, and $\mathrm{NF}-\kappa \mathrm{B}$ has been reported to serve notable roles in the development of lung injury $(30,31)$. These data indicate that miR-15a-5p/TNIP2 may serve critical roles in acute lung injury.

Therefore, the purpose of the present study was to investigate the expression of miR-15a-5p in THS induced acute lung injury and the molecular mechanism underlying its role.

\section{Materials and methods}

Clinical samples. A total of 30 peripheral blood samples (5 ml per individual) from 30 patients (median age, 38.2; age range, 24-59 years; 25 male; 5 female) with acute lung injury induced by trauma-hemorrhagic shock (THS), as well as 30 peripheral blood samples from 30 healthy volunteers (median age, 37.1; age range, 21-58 years old; 25 male; 5 female) were collected at Affiliated Hospital of Jiangsu University (Zhenjiang, China) between June 2015 and June 2017. Blood samples were collected from patients $24 \mathrm{~h}$ after traumatic hemorrhagic shock and stored at $-80^{\circ} \mathrm{C}$. Informed consent was signed for each patient participating in the study. The present study was approved by the Ethics Committee of Affiliated Hospital of Jiangsu University.

Cell culture. 293T cells were purchased from American Type Culture Collection. Cells were grown in DMEM (Gibco; Thermo Fisher Scientific, Inc.) containing 10\% fetal bovine serum (FBS; Gibco; Thermo Fisher Scientific, Inc.) and $1 \%$ streptomycin-penicillin solution and incubated at $37^{\circ} \mathrm{C}$ with $5 \% \mathrm{CO}_{2}$.

Animals and THS model establishment. A total of 50 male Sprague-Dawley rats (10-14 weeks old; 360-400 g) were obtained from Vital River Company (Beijing, China). Rats were housed at $25 \pm 5^{\circ} \mathrm{C}, 50 \%$ humidity and $12 \mathrm{~h}$ dark/light cycle conditions. All rats had free access to food and water. The present experiments were conducted following the Recommended Guideline for the Care and Use of Laboratory Animals issued by Chinese Council on Animal Research. The current study was approved by Animal Ethics Committee of the Affiliated Hospital of Jiangsu University.

The rat model of THS was conducted according to a previous study (32). Rats were randomly assigned into five groups: Control (Sham; rats underwent the same anesthetic and surgical procedures, but trauma/hemorrhage was not induced), THS, THS + inhibitor control (intraperitoneal injection), THS + miR-15a-5p inhibitor (intraperitoneal injection), THS + miR-15a-5p inhibitor + TNIP2-siRNA (intraperitoneal injection). Rats were intraperitoneally injected with inhibitor control (80 mg/kg/day; 5'-CAGUACUUUUGUGUAGUACAA-3'; Shanghai GenePharma Co., Ltd.), miR-15a-5p inhibitor
(80 mg/kg/day; 5'-CACUGGUACAAGGGUUGGGAGA-3'; Shanghai GenePharma Co., Ltd.) or miR-15a-5p inhibitor $(80 \mathrm{mg} / \mathrm{kg} / \mathrm{day})+$ TNIP2-siRNA $(80 \mathrm{mg} / \mathrm{kg} / \mathrm{day}$; cat. no. sc-44638; Santa Cruz Biotechnology, Inc.) prior to surgery using in vivo transfection reagent (EntransterTM-in vivo; Engreen Biosystem Co., Ltd.). Rats in the Sham and THS groups were given saline $(0.9 \% \mathrm{NaCl})$ solution intraperitoneally. All rats were anesthetized with $30 \mathrm{mg} / \mathrm{kg}$ pentobarbital and handled $24 \mathrm{~h}$ after TSH induction. After the specified treatments, following experiments were conducted.

Evan's blue dye (EBD). EBD was performed to evaluate the lung permeability according to a previous study (33). In brief, $1 \mathrm{ml}$ of $1 \%$ EBD solution was injected into rats from different groups via the jugular vein. Then, $1.5 \mathrm{ml}$ blood sample was collected from the femoral artery catheter. After $20 \mathrm{~min}$, the rats were sacrificed and the lungs were removed before being washed three times with $5 \mathrm{ml}$ of physiological saline to collect bronchoalveolar lavage fluid (BALF). The supernatant was collected via centrifugation $(1,000 \mathrm{x}$ g for $10 \mathrm{~min}$ at $4^{\circ} \mathrm{C}$ ). The concentration of EBD in plasma and BALF was detected via measuring the absorbance value at $620 \mathrm{~nm}$ using a micro-plate reader (Elx800; BioTek Instruments, Inc.). Finally, the ratio of EBD in the BALF to EBD in plasma was calculated.

Lung permeability index. To calculate the lung permeability index, the ratio of the BALF protein concentration to the serum protein concentration was measured, according to a previous study (34). Briefly, $1 \mu \mathrm{l}$ of sample supernatant was mixed with $4 \mu \mathrm{l}$ normal saline and $250 \mu \mathrm{l}$ Coomassie brilliant blue G250 at room temperature for $5 \mathrm{~min}$. The absorbance at $595 \mathrm{~nm}$ was measured using a micro-plate reader (BioTek Instruments, Inc.). Calculation of protein concentration in BALF and plasma was based on a standard curve of bovine serum albumin (BSA).

Lung edema evaluation. Lung edema was assessed by measuring lung wet/dry weight ratio (W/D) of the rats. The rats were anesthetized with $3 \%$ isoflurane. Then the lungs from rats in different groups were removed and the wet weight (W) of rats were measured. After drying for $72 \mathrm{~h}$ at $100^{\circ} \mathrm{C}$, the dry weight (D) of the lungs from rats in different groups was also detected. Finally, the ratio of W/D was calculated.

Measurement of proinflammatory factors. Peripheral blood samples were centrifuged at $1,000 \times \mathrm{g}$ for $10 \mathrm{~min}$ at $4^{\circ} \mathrm{C}$ and the serum was collected. Bronchoalveolar lavage fluid (BALF) was collected by intratracheal instillation of lungs with sterile PBS three times, and then the BALF supernatant was harvested via centrifugation at $800 \mathrm{x}$ for $10 \mathrm{~min}$ at $4^{\circ} \mathrm{C}$. ELISA kits were then used to measure the levels of pro-inflammatory factors including tumor necrosis factor (TNF)- $\alpha$ (cat. no. PT516; Beyotime Institute of Biotechnology) and interleukin (IL)-6 (cat. no. PI328; Beyotime Institute of Biotechnology), in serum or BALF, according to the manufacturer's instructions of each kit.

Nitric oxide $(\mathrm{NO})$ detection. The lung tissues from rats of different groups were homogenized, freeze-thawed with liquid 
nitrogen three times and then centrifuged $(10,000 \times \mathrm{g} ; 10 \mathrm{~min}$; $4^{\circ} \mathrm{C}$ ) to collect supernatants. Bicinchoninic Acid Protein Assay kit was used to detect protein concentrations, according to the manufacturer's instructions. Then, proteins were diluted to $2 \mu \mathrm{g} / \mu \mathrm{l}$ in PBS. Finally, total Nitric Oxide Assay kit (cat. no. S0023; Beyotime Institute of Biotechnology) was used to measure the concentration of $\mathrm{NO}$, following the manufacturer's instructions.

Reverse transcription-quantitative PCR (RT-qPCR). Total RNA from tissues or blood samples was isolated using TRIzol reagent (Invitrogen; Thermo Fisher Scientific, Inc.) and reversely transcribed into cDNA with the PrimeScript ${ }^{\mathrm{TM}}$ RT reagent kit (Takara Bio, Inc.) as per the manufacture's protocol. SYBR $^{\circledR}$ Premix Ex Taq ${ }^{\mathrm{TM}}$ II (Takara Bio Inc.) was used for qPCR analysis. Amplification conditions for qPCR were as follows: $10 \mathrm{~min}$ at $95^{\circ} \mathrm{C}$, followed by 35 cycles of $15 \mathrm{sec}$ at $95^{\circ} \mathrm{C}$ and $40 \mathrm{sec}$ at $55^{\circ} \mathrm{C}$. U6 for miRNA (24) and GAPDH for mRNA (28) were used as the endogenous controls. The primer sequences were as follows: miR-15a-5p forward, 5'-GG GTAGCAGCACATAATGGTTTGTG-3' and reverse, 5'-CAG TGCGTGTCGTGGAGT-3'; U6 forward, 5'-GCTTCGGCA GCACATATACTAAAAT-3' and reverse, 5'-CGCTTCACG AATTTGCGTGTCAT-3'; GAPDH forward, 5'-CTTTGG TATCGTGGAAGGACTC-3' and reverse, 5'-GTAGAGGCA GGGATGATGTTCT-3'; TNIP2 forward, 5'-CTAAAGAGG CGGCAGGTCCCTC-3' and reverse, 5'-CAAGATGACCTT CCAGTGAC-3'; iNOS forward, 5'-TCTCCGACCACCACT ACAGCAA-3' and reverse: 5'-GGGGAACTGGGCAGA CTCAA-3'. Relative gene expression was quantified using the $2^{-\Delta \Delta \mathrm{Cq}}$ method (35).

Western blot assay. Total proteins from tissues/blood samples were extracted using RIPA lysis buffer (50 mM Tris ( $\mathrm{pH} 7.4)$, $150 \mathrm{mM} \mathrm{NaCl}, 1 \% \mathrm{NP}-40,0.5 \%$ sodium deoxycholate) supplemented with PMSF at a final concentration of $1 \mathrm{mM}$. BCA protein assay kit was used to detect the concentrations of proteins. Protein samples ( $25 \mu \mathrm{g}$ per lane) were separated using 12\% SDS-PAGE, transferred onto PVDF membranes (EMD Millipore), and blocked using 5\% skimmed milk at room temperature for $1 \mathrm{~h}$. Then, the membranes were incubated with primary antibodies: TNIP2, phosphorylated (p-)NF-кB (p-p65), inducible nitric oxide synthase (iNOS) and $\beta$-actin (1:1,000; Cell Signaling Technology, Inc.) at $4^{\circ} \mathrm{C}$ overnight. Subsequently, the membranes were incubated with the anti-rabbit IgG HRP-linked antibody (cat. no. 7074; 1:5,000; Cell Signaling Technology, Inc.) at room temperature for 2 h. Finally, ECL reagents (EMD Millipore) were used to visualize the corresponding protein bands. The band densities of p-p65 was analyzed using Gel-Pro-Analyzer software (Version 6.3; Media Cybernetics, Inc.), and the relative protein level of p-p65 was presented as fold of the control group.

Dual luciferase reporter assay. In the current study, TargetScan bioinformatics software version 7.1 (www. targetscan.org/vert_71) was used to predict the targets of miR-15a-5p, and it was revealed that TNIP2 was a potential target of miR-15a-5p. To confirm the binding sites between miR-15a-5p and TNIP2, the wild type (WT-TNIP2) and mutant (MUT-TNIP2) 3'UTR of TNIP2 were cloned into a pmiR-RB-ReportTM dual luciferase reporter gene plasmid vector (Guangzhou RiboBio Co., Ltd). Subsequently, 293 T cells were cotransfected with WT-TNIP2 or MUT-TNIP2 and miR-15a-5p mimic or mimic control using Lipofectamine $^{\circledR} 2000$ (Invitrogen; Thermo Fisher Scientific, Inc.), as per the manufacturer's protocols. After $48 \mathrm{~h}$ of cell transfection, luciferase activity was determined using the dualluciferase assay system (Promega Corporation) in line with the manufacturer's protocol. Luciferase activity was normalized to Renilla luciferase activity.

Statistical analysis. Data obtained from the current study was displayed as the mean \pm SD. SPSS 16.0 statistical software (SPSS, Inc.) was used for all statistical analyses. Unpaired Student's t-tests and one-way ANOVA followed by Tukey's post hoc test were performed to analyze the differences between groups. $\mathrm{P}<0.05$ was considered to indicate a statistically significant difference.

\section{Results}

miR-15a-5p is significantly upregulated in patients with THS and rats. To detect the level of miR-15a-5p in the blood of patients with THS, RT-qPCR was performed. As revealed in Fig. 1A, compared with the healthy control, the level of miR-15a-5p was significantly increased in the blood samples of patients with THS. The findings indicated that miR-15a-5p may influence the development of THS.

Subsequently, the levels of miR-15a-5p were detected in the blood and the lung tissues of THS rats, and the results showed that miR-15a-5p was significantly upregulated in the blood and the lung tissues of THS rats (Fig. 1B and C).

TNIP2 is a target of miR-15a-5p. To investigate the target genes of miR-15a-5p, we firstly predicted the target gene of miR-15a-5p using TargetScan (www.targetscan. org/vert_71). The results indicated that miR-15a-5p has hundreds of potential target genes, including TNIP2. The role of TNIP2 in THS-induced lung injury is yet to be fully elucidated. Therefore, TNIP2 was selected for further study. Subsequently, to reveal whether miR-15a-5p directly binds TNIP2, a dual luciferase reporter assay was performed. The miR-15a-5p-TNIP2-WT or miR-15a-5p-TNIP2-MUT reporter plasmid were co-transfected into $293 \mathrm{~T}$ cells with miR-15a-5p mimic or mimic control and it was revealed that the luciferase activity was significantly decreased in the 293T cells co-transfected with miR-15a-5p mimic with miR-15a-5p-TNIP2-WT, but not with miR-15a-5p-TNIP2-MUT (Fig. 2). The data suggested that TNIP2 represents a target gene of miR-15a-5p.

TNIP2 is significantly downregulated in patients with THS and rats. To detect the expression of TNIP2 in the blood of patients with THS and rats, RT-qPCR and western blot assay were performed. As indicated in Fig. 3A and B, compared with the healthy control, the mRNA and protein levels of TNIP2 significantly decreased in the blood samples of patients with THS. Then the mRNA and protein levels of TNIP2 were detected in the blood and the pulmonary tissues of rats with 

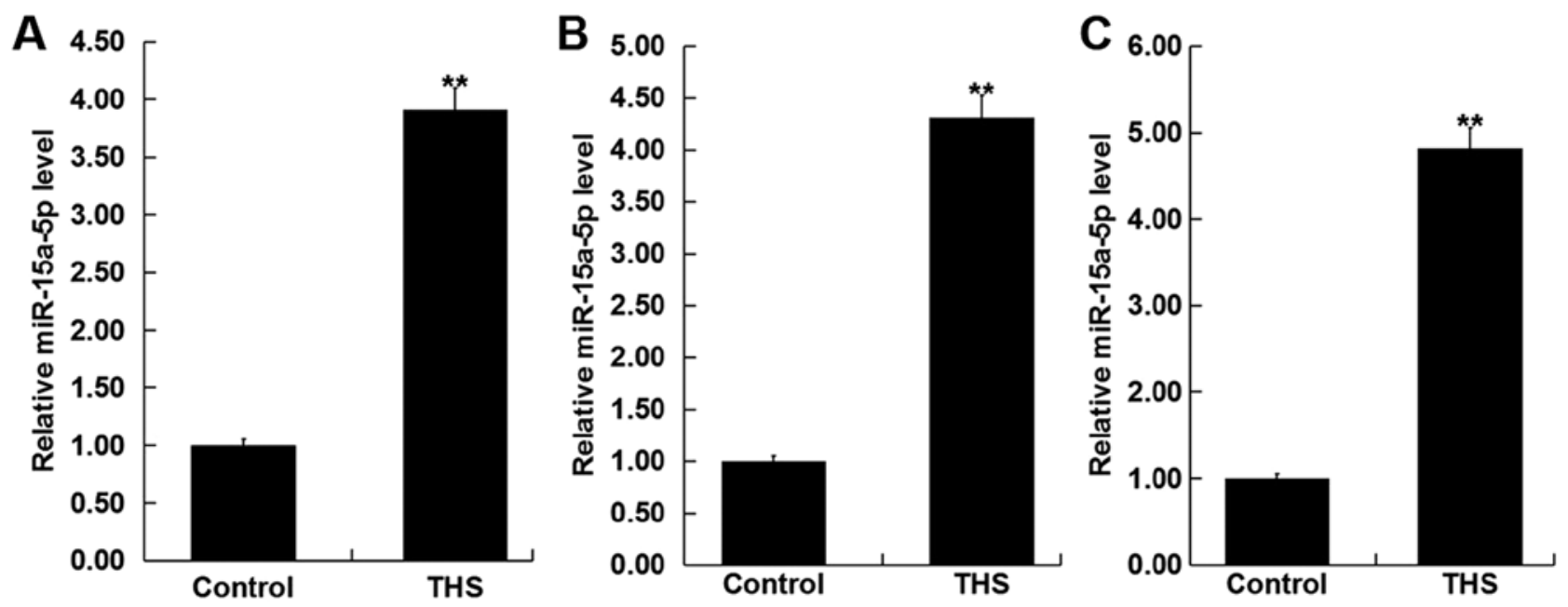

Figure 1. Expression level of miR-15a-5p in patients with THS and the rat model. (A) Expression level of miR-15a-5p in the blood of patients with THS was detected using RT-qPCR. The level of miR-15a-5p in the (B) blood and (C) lung tissues of THS rats was detected using RT-qPCR. Data were expressed as mean \pm SD. ${ }^{* *} \mathrm{P}<0.01$ vs. control group. miR, microRNA; THS, traumatic hemorrhagic shock; RT-qPCR, reverse transcription-quantitative PCR.

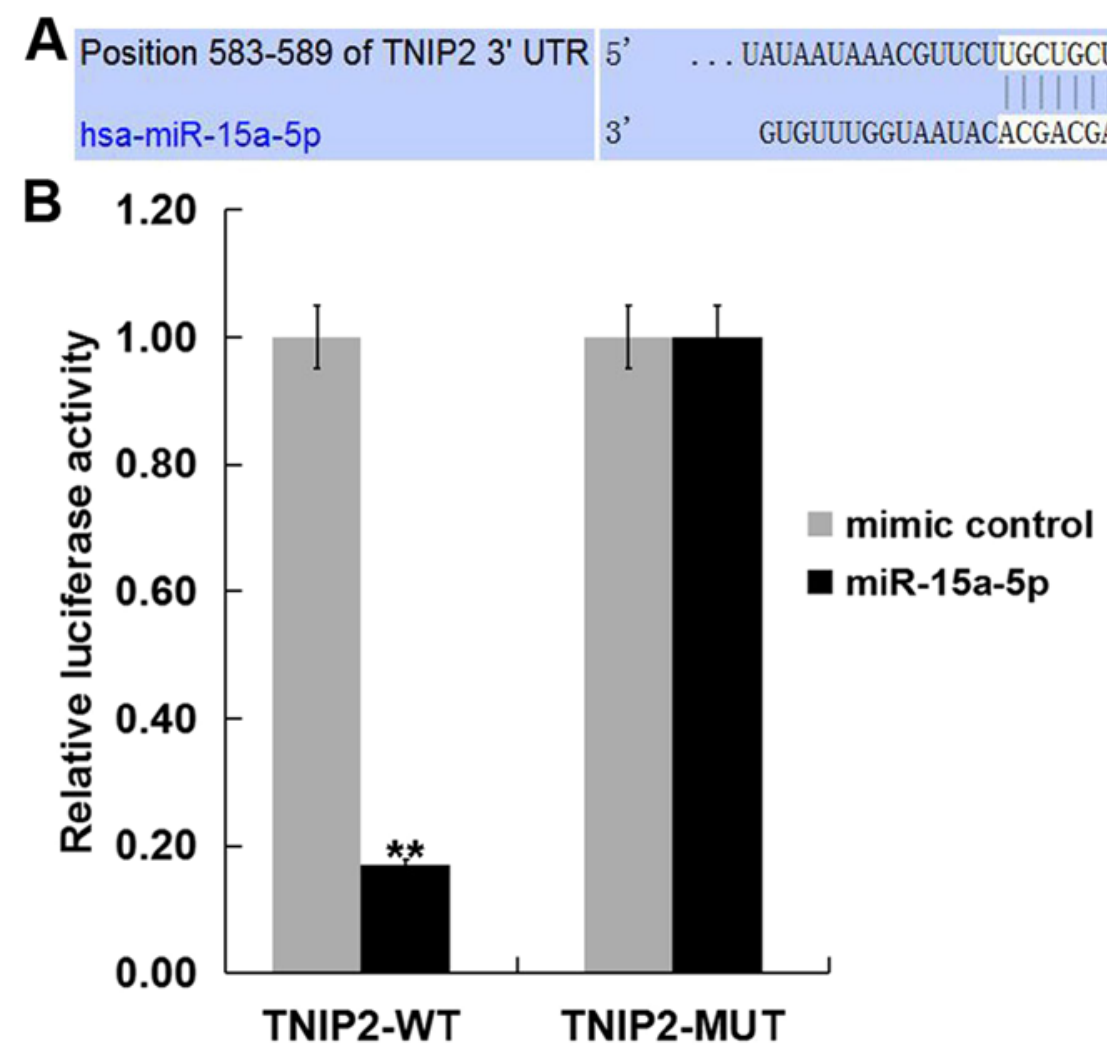

Figure 2. TNIP2 is a direct target of miR-15a-5p. (A) Interaction between miR-15a-5p and 3'UTR of TNIP2 was predicted using TargetScan. (B) Luciferase activity of a reporter containing a wild-type TNIP2 3'UTR or a mutant TNIP2 3' UTR are presented. TNIP2-MUT indicates the TNIP2 3' UTR with a mutation in the miR-15a-5p binding site. All data are presented as the mean $\pm \mathrm{SD}$ of three independent experiments. ${ }^{* *} \mathrm{P}<0.01 \mathrm{vs}$. mimic control. miR, microRNA; UTR, untranslated region; THS, traumatic hemorrhagic shock; WT, wild type; TNIP2, TNFAIP3-interacting protein 2.

THS, and the results revealed that TNIP2 was significantly downregulated in the blood and the pulmonary tissues of THS rats (Fig. 3C-E).

miR-15a-5p inhibitor alleviates lung injury in THS rats. To investigate the effect of miR-15a-5p on THS rats, rats were intraperitoneally injected with inhibitor control or miR-15a-5p inhibitor, prior to THS induction. After $24 \mathrm{~h}$, it was revealed that the miR-15a-5p inhibitor significantly decreased the level of miR-15a-5p in the blood and lung tissues of THS rats (Fig. 4A and B). Moreover, TNIP2-siRNA significantly decreased the mRNA level of TNIP2 in the blood and lung tissues of THS rats (Fig. 4C and D). The miR-15a-5p inhibitor significantly increased both the mRNA and protein levels of TNIP2 in the blood and the lung tissues of THS rats (Fig. 4E-H). 


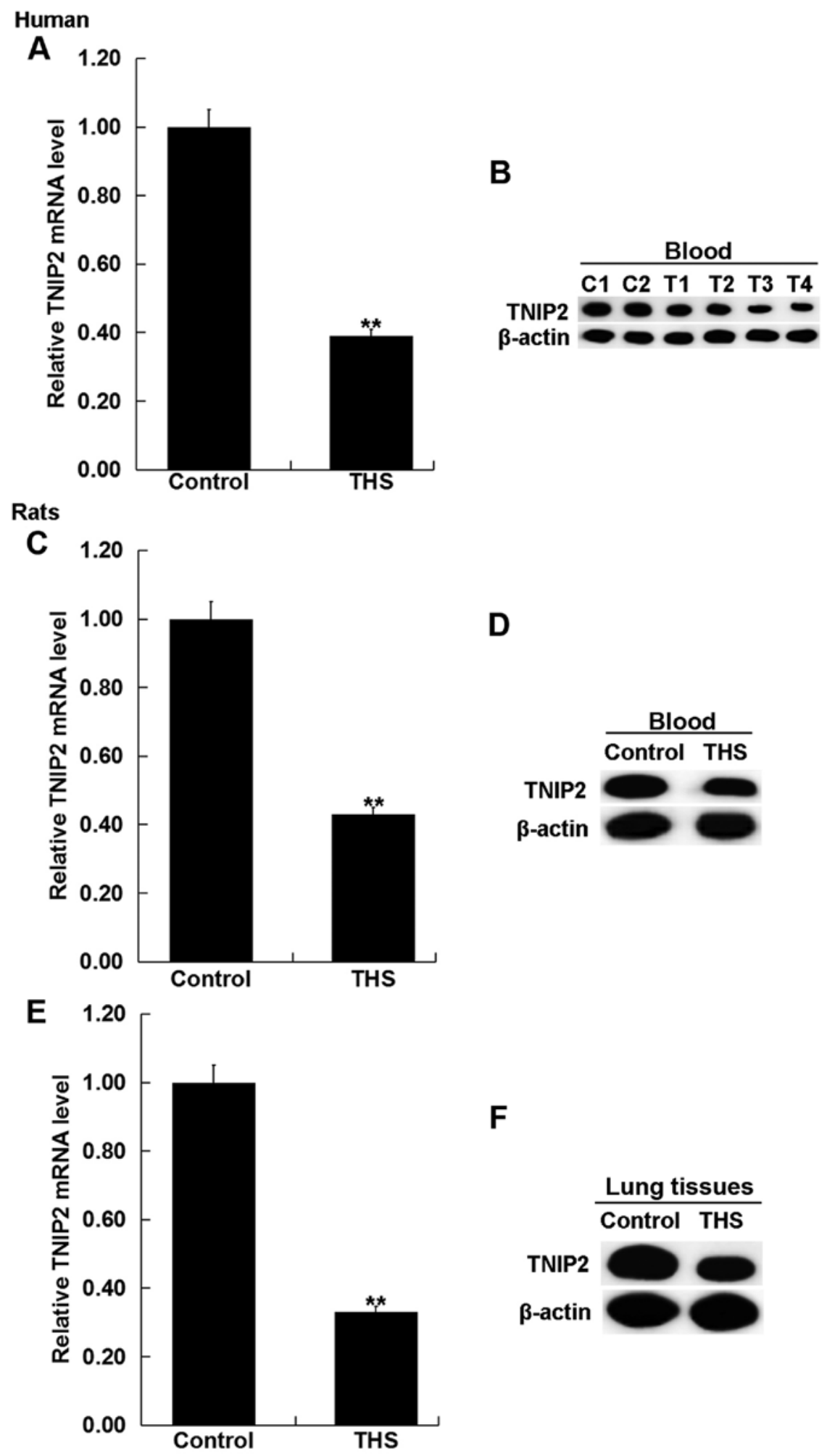

Figure 3. Expression level of TNIP2 in patients with THS and rats. (A) mRNA and (B) protein level of TNIP2 in the blood of patients with THS was detected using RT-qPCR and western blotting (C1, C2: Healthy control; T1-T4: Patients with THS). (C) mRNA and (D) protein level of TNIP2 in the blood of THS rats was detected using RT-qPCR and western blotting. (E) mRNA and (F) protein level of TNIP2 in the lung tissues of THS rats was detected using RT-qPCR and western blotting. Data were expressed as mean $\pm \mathrm{SD} .{ }^{* *} \mathrm{P}<0.01$ vs. control group. THS, traumatic hemorrhagic shock; TNIP2, TNFAIP3-interacting protein 2; RT-qPCR, reverse transcription-quantitative PCR.

The effects of miR-15a-5p on THS-induced lung injury were subsequently investigated. The ratio of EBD in the BALF to EBD in plasma of THS rats, and the lung permeability index (ratio of the BALF protein concentration to the serum protein concentration) were measured to determine lung capillary permeability. The results demonstrated that these two indicators were significantly increased after THS but were decreased by miR-15a-5p inhibitor treatment. However, the ratio of EBD in the BALF to EBD in plasma of THS rats and the lung permeability index were significantly 
A

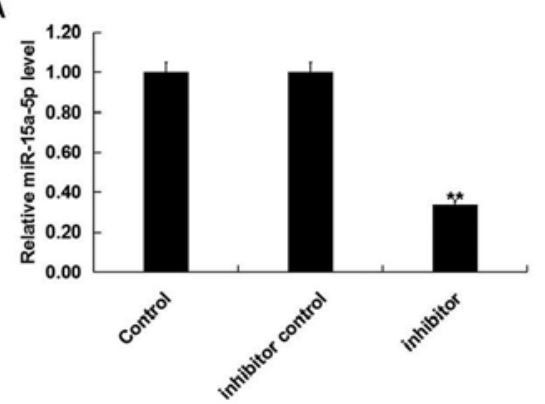

D
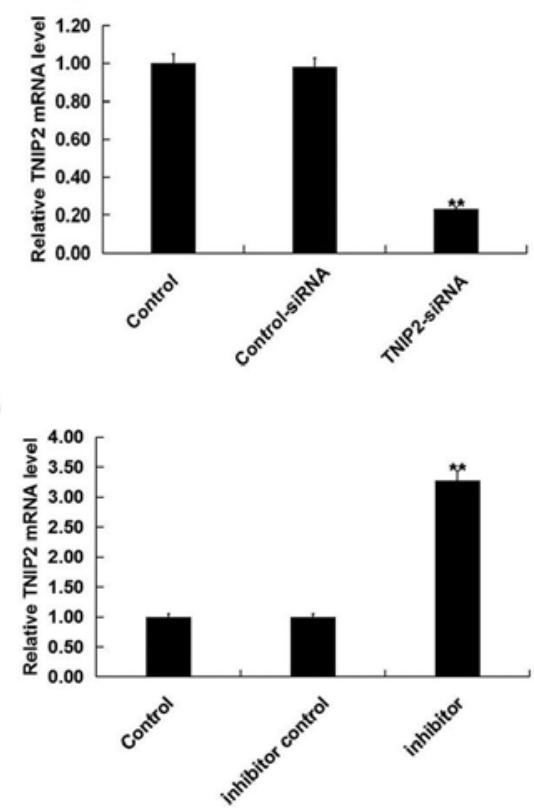

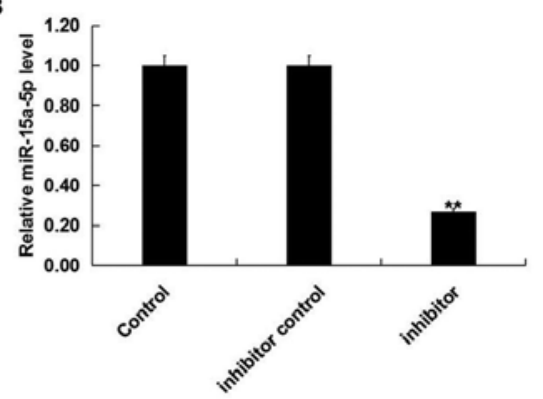

E

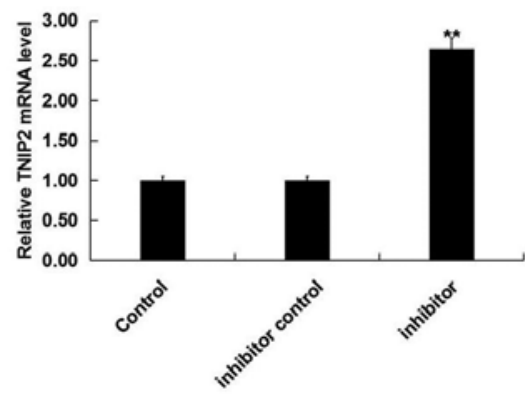

$\mathrm{H}$

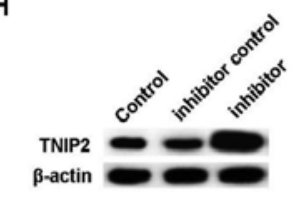

c
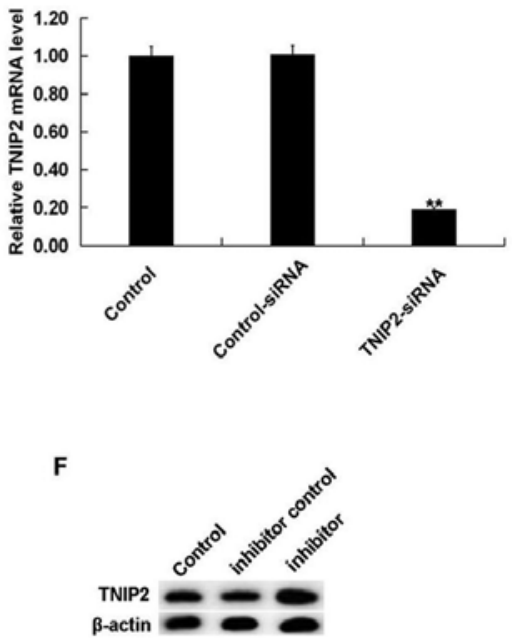

Figure 4. Effect of miR-15a-5p on TNIP2 expression in THS rats. Rats were intraperitoneally injected with inhibitor control, or miR-15a-5p inhibitor prior to THS induction. After $24 \mathrm{~h}$, the level of miR-15a-5p and TNIP2 in the blood and lung tissues of THS rats was determined. The level of miR-15a-5p in the (A) blood and (B) lung tissue of THS rats was detected using RT-qPCR. The TNIP2 mRNA level in the (C) blood and (D) lung tissue of THS rats was detected using RT-qPCR: The mRNA and protein level of TNIP2 in the blood of THS rats was detected using (E) RT-qPCR and (F) western blotting. The mRNA and protein level of TNIP2 in the lung tissues of THS rats was detected using (G) RT-qPCR and (H) western blotting. Data were expressed as mean \pm SD. ${ }^{* *} \mathrm{P}<0.01$ vs. control group. miR, microRNA; THS, traumatic hemorrhagic shock; TNIP2, TNFAIP3-interacting protein 2; RT-qPCR, reverse transcription-quantitative PCR.

enhanced by transfection with TNIP2-siRNA compared with the miR-15a-5p inhibitor treatment group (Fig. 5A and B). Moreover, the lung W/D ratio was calculated to determine the lung edema in current study, and it was revealed that the increased lung wet/dry ratio induced by THS was decreased by miR-15a-5p inhibitor treatment, and this reduction was eliminated by TNIP2-siRNA (Fig. 5C). Taken together, the data indicated that miR-15a-5p inhibitor alleviated lung injury in THS rats.

miR-15a-5p inhibitor prevents inflammatory response in THS rats. It was determined whether the miR-15a-5p inhibitor had anti-inflammatory effects. As depicted in Fig. 6, the increased levels of TNF- $\alpha$ and IL-6 in BALF and serum of THS rats were significantly decreased following miR-15a-5p inhibitor treatment, and these reductions were eliminated by TNIP2-siRNA.

miR-15a-5p inhibitor decreases the expression of NO and iNOS in THS rats. Overproduction of NO by iNOS influences the pathogenesis of THS-induced lung injury (36). Thus, the present study detected the concentration of NO in the lungs of THS rats and the mRNA and protein levels of iNOS were determined. It was demonstrated that the concentration of NO and protein and mRNA levels of iNOS in THS rats significantly increased compared with the sham group. However, the miR-15a-5p inhibitor notably decreased the expression of NO and iNOS in the lung tissues of THS rats, and these decreases were attenuated by TNIP2-siRNA (Fig. 7).

miR-15a-5p inhibitor decreases $N F-\kappa B$ activation in THS rats. Finally, to investigate the mechanism of the effect of miR-15a-5p inhibitor on THS rats, the NF- $\kappa$ B pathway was analyzed by determining p-p65 protein expression and the ratio of p-p65/p65 expression. It was revealed that the enhanced p-p65 protein level (Fig. 8A) and increased p-p65/p65 ratio (Fig. 8B) in lung tissues of THS rats was decreased following treatment with the miR-15a-5p inhibitor, and this reduction was alleviated by TNIP2-siRNA (Fig. 8). 

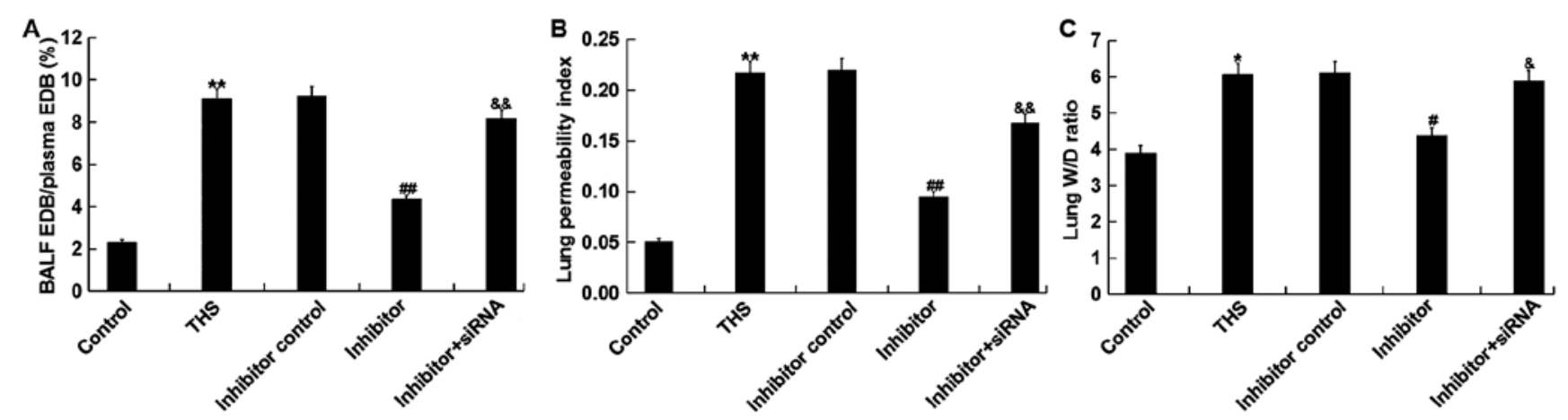

Figure 5. Effect of miR-15a-5p on lung injury induced by THS in rats. (A) Ratio of the percentage of EBD in the BALF to EBD in the plasma. (B) Lung permeability index $=$ BALF protein concentration/plasma protein concentration. (C) Lung W/D weight ratio. Data are expressed as mean $\pm \mathrm{SD}$. ${ }^{*} \mathrm{P}<0.05,{ }^{* *} \mathrm{P}<0.01 \mathrm{vs}$. control group; ${ }^{~} \mathrm{P}<0.05 ;{ }^{\# \#} \mathrm{P}<0.01$ vs. THS group; ${ }^{\&} \mathrm{P}<0.05$, ${ }^{\&}{ }^{\&} \mathrm{P}<0.01$ vs. inhibitor group. miR, microRNA; THS, traumatic hemorrhagic shock; siRNA, small interfering RNA; EBD, Evan's blue dye; BALF, bronchoalveolar lavage fluid; W, wet; D, dry.
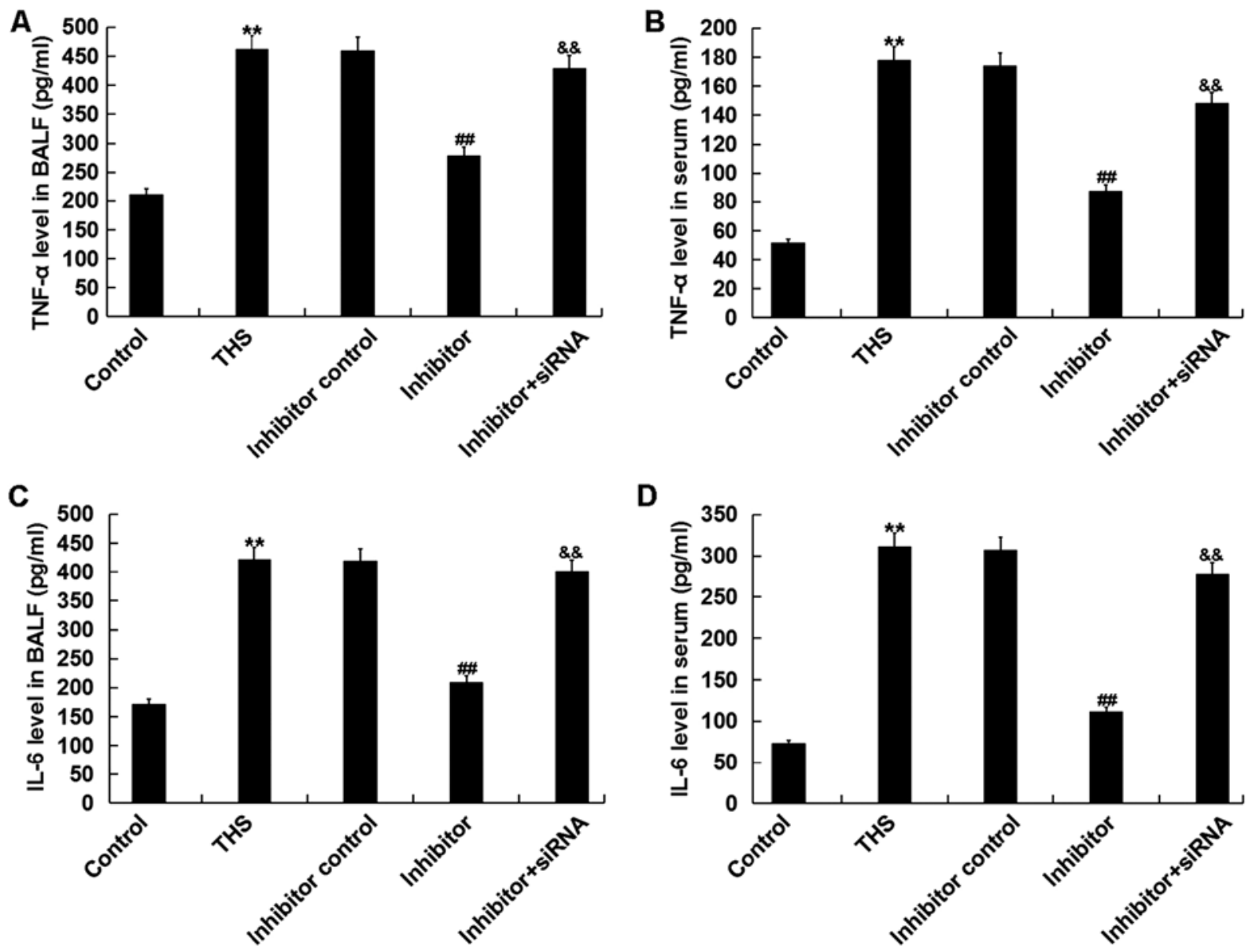

D

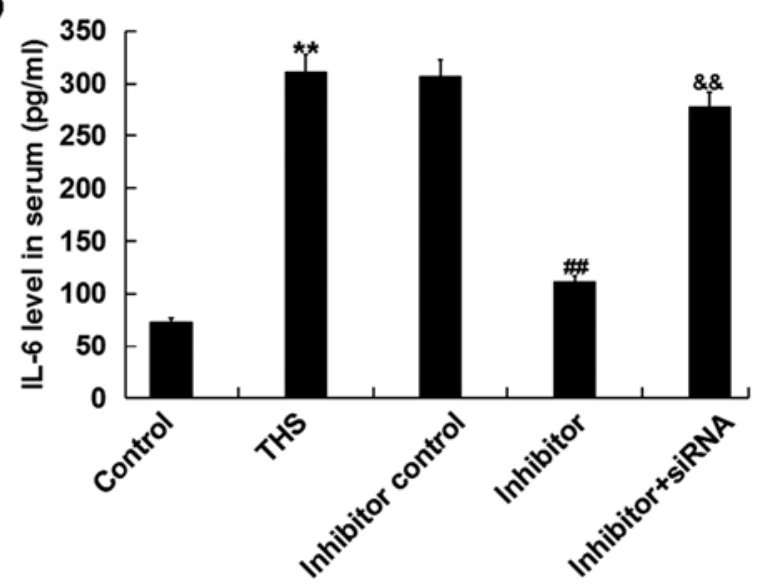

Figure 6. Effect of miR-15a-5p on pro-inflammatory factors production in THS rats. The levels of TNF- $\alpha$ in (A) BALF and (B) serum and the levels of IL-6 in (C) BALF and (D) serum in rats were detected using ELISA. Data are expressed as mean $\pm \mathrm{SD}$. ${ }^{* *} \mathrm{P}<0.01$ vs. control group; ${ }^{\# / \#} \mathrm{P}<0.01$ vs. THS group; \&\& $\mathrm{P}<0.01$ vs. inhibitor group. miR, microRNA; THS, traumatic hemorrhagic shock; TNIP2, TNFAIP3-interacting protein 2; BALF, bronchoalveolar lavage fluid; IL, interleukin; TNF- $\alpha$, tumor necrosis factor- $\alpha$; siRNA, small interfering RNA.

\section{Discussion}

The present study demonstrated that miR-15a-5p was significantly upregulated in patients and rats with acute lung injury induced by THS. TNIP2 was revealed as a target of miR-15a-5p, and it was downregulated in patients and rats with THS. Further analyses indicated that downregulation of miR-15a-5p significantly relieved THS-induced acute lung injury and inhibited the inflammatory response in a rat model of THS via targeting TNIP2. Moreover, it was revealed that THS-induced NO production, iNOS expression and NF- $\mathrm{KB}$ pathway activation in lung tissues were all repressed following miR-15a-5p inhibition via targeting TNIP2, indicating that these pathways may be a part of the mechanisms responsible 


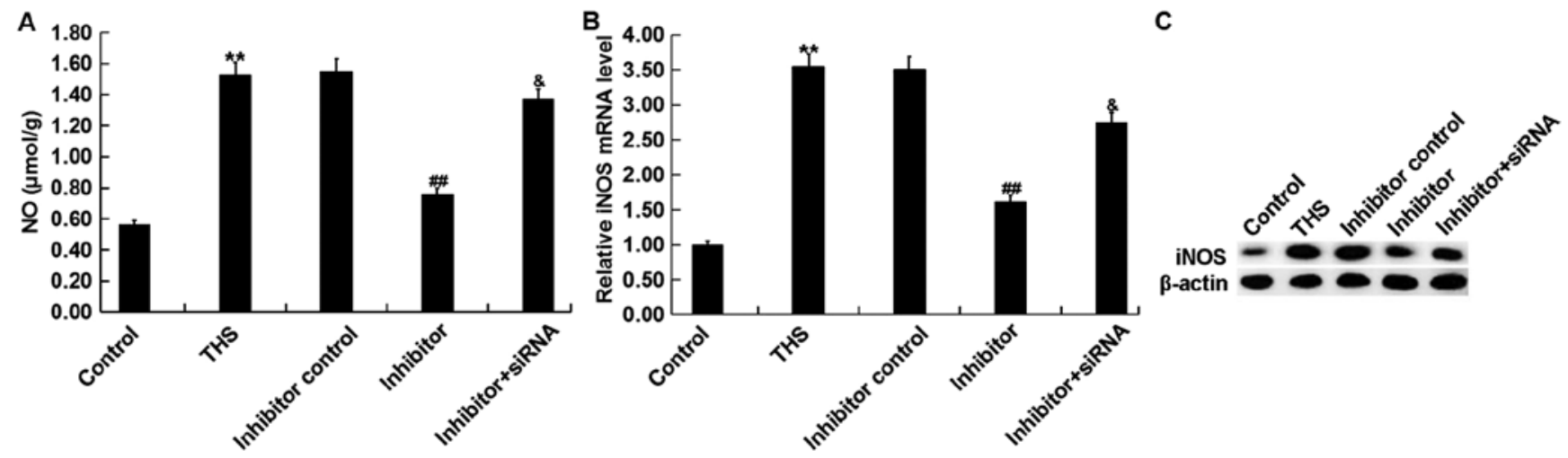

Figure 7. Effect of miR-15a-5p on NO release and iNOS expression in THS rats. (A) Lung NO concentrations were detected using a commercial kit. The mRNA and protein level of iNOS in lung tissues of rats was detected using (B) reverse transcription-quantitative PCR and (C) western blotting, respectively. Data were expressed as mean $\pm \mathrm{SD}$. ${ }^{* *} \mathrm{P}<0.01$ vs. control group; ${ }^{\# \#} \mathrm{P}<0.01$ vs. THS group; ${ }^{\circledR} \mathrm{P}<0.05$ vs. inhibitor group. NO, nitric oxide; iNOS, inducible nitric oxide synthase; THS, traumatic hemorrhagic shock; siRNA, small interfering RNA; miRNA, microRNA.

A

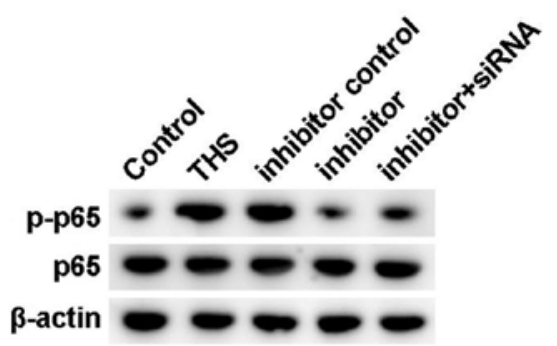

B

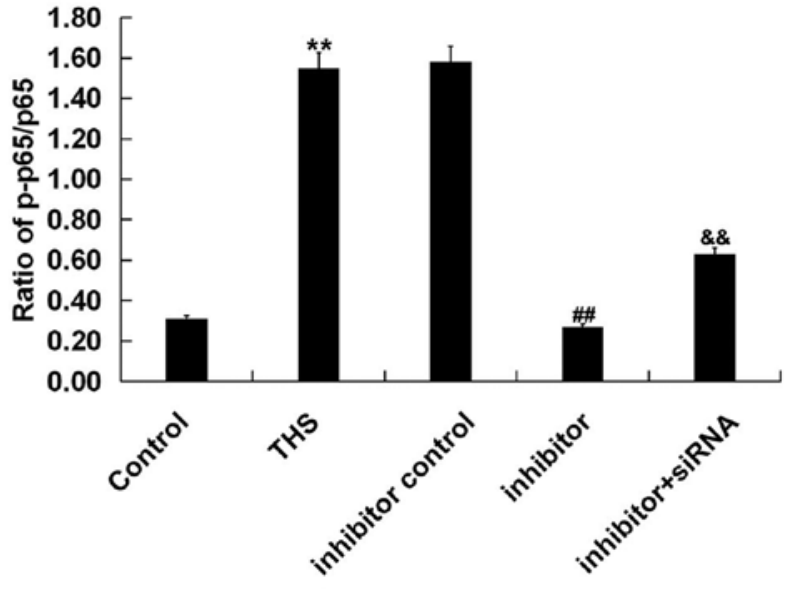

Figure 8. Effect of miR-15a-5p on NF- $\kappa$ B activation in THS rats. The protein level of p-p65 and p65 was measured using (A) western blot assay and (B) the ratio of p-p65/p65 was calculated and presented (B). Data were expressed as mean $\pm \mathrm{SD} .{ }^{* *} \mathrm{P}<0.01$ vs. control group; ${ }^{\# \#} \mathrm{P}<0.01$ vs. THS group; ${ }^{\& \&} \mathrm{P}<0.01$ vs. inhibitor group. miRNA, microRNA; p-, phosphorylated; THS, traumatic hemorrhagic shock; siRNA, small interfering RNA.

for the protective effects of miR-15a-5p downregulation on lungs in THS rats.

THS has a very high mortality rate, and acute lung injury is one of the major complications of THS $(1,2,5)$. Since the molecular mechanisms underlying acute lung injury after THS are very complicated, there is still no effective method for treating THS-induced acute lung injury. Previous studies on miRNAs have provided new directions for the diagnosis and treatment of various diseases (16-22). However, there have been few studies on miRNAs in THS-induced acute lung injury. The present study investigated the functional role of miR-15a-5p in THS-induced acute lung injury and explored the underlying molecular mechanism.

miR-15a-5p had not been well characterized but has been studied in several cancer types, including endometrial cancer, chronic myeloid leukemia, colorectal adenocarcinoma and hepatocellular carcinoma (23-26). The present study investigated whether miR-15a-5p was involved in THS induced acute lung injury. Firstly, the level of miR-15a-5p was determined in the blood of patients with THS and in the blood and the lung tissues of THS rats, and the results revealed that miR-15a-5p was highly expressed in both the blood of patients with THS and the blood and lung tissues of THS rats, indicating the involvement of miR-15a-5p in THS-induced acute lung injury. Then, to explore the potential roles of miR-15a-5p in THS-induced acute lung injury, the targets of miR-15a-5p were predicted, and it was revealed that TNFAIP3-interacting protein 2 (TNIP2) was a direct target of miR-15a-5p and it was downregulated in patients with THS and rats.

The protein encoded by the TNIP2 gene inhibits the activation of the $\mathrm{NF}-\kappa \mathrm{B}$ pathway (27). It is generally believed that TNIP2 serves an important regulatory role in the $N F-\kappa B$ signaling pathway $(28,29)$. NF- $\kappa \mathrm{B}$ is an important transcription factor involved in the regulation of survival, immune, oxidative stress, and inflammatory reaction (37-39). Therefore, it was hypothesized that miR-15a-5p may serve a role in THS-induced acute lung injury by regulating the TNIP2/NF- $\kappa \mathrm{B}$ signaling pathway and thereby regulating the inflammatory response. As expected, the findings of current study indicated that downregulation of miR-15a-5p significantly relieved THS induced lung injury, inhibited inflammatory response in lung tissues of THS rats by targeting TNIP2. Besides, miR-15a-5p inhibitor 
significantly reduced the protein level of p-p65 and the ratio of p-p65/p65 in the lung tissue of THS rats, indicating the inhibition of NF- $\kappa \mathrm{B}$ signaling pathway, and this effect was reversed by TNIP2-siRNA. Overproduction of NO by iNOS is involved in the pathogenesis of THS-induced lung injury (37). The current study also revealed the inhibitory effect of miR-15a-5p inhibitor on NO production and iNOS expression in the lung tissues of THS rats. However, a group of miR-15a-5p inhibitor + control-siRNA was not set up in our experiments, and this may represent a limitation of the present study.

Taken together, the current results demonstrated that miR-15a-5p was upregulated in THS-induced acute lung injury, and its inhibition served a protective role in via repressing the inflammatory response by regulating the TNIP2/NF- $\mathrm{B}$ signaling pathway. miR-15a-5p may represent a potential diagnostic marker and therapeutic target for the treatment of THS-induced acute lung injury. However, the current study was a preliminary study of the role of miR-15a-5p in the pathogenesis of acute lung injury induced by THS, and this topic requires extensive research. It would be beneficial to determine the correlation of miR-15a-5p expression with the clinical characteristics and prognosis of patients with THS in order to elucidate the role of miR-15a-5p in THS-induced lung injury. Furthermore, there is a need to demonstrate the role of TNIP2 in THS-induced lung injury in future studies.

\section{Acknowledgements}

Not applicable.

\section{Funding}

The present study was supported by Zhenjiang social development project (grant no. SH2017025).

\section{Availability of data and materials}

The datasets used and/or analyzed during the current study are available from the corresponding author on reasonable request.

\section{Authors' contributions}

FZ contributed to study design, data collection, statistical analysis, data interpretation and manuscript preparation. ZZL, ZJM, FXW and CS contributed to data collection and statistical analysis. HZC performed experiments, and contributed to manuscript preparation and literature searching. All authors read and approved the final manuscript.

\section{Ethics approval and consent to participate}

Informed consent was signed for each patient participating in the study. The present study was approved by the Ethics Committee of Affiliated Hospital of Jiangsu University.

\section{Patient consent for publication}

All patients consented to publication.

\section{Competing interests}

The authors declare that they have no competing interests.

\section{References}

1. Kauvar DS, Lefering R and Wade CE: Impact of hemorrhage on trauma outcome: An overview of epidemiology, clinical presentations, and therapeutic considerations. J Trauma 60 (Suppl 6): S3-S11, 2006.

2. Kauvar DS and Wade CE: The epidemiology and modern management of traumatic hemorrhage: US and international perspectives. Crit Care 9 (Suppl 5): S1-S9, 2005.

3. Angele MK, Schneider CP and Chaudry IH: Bench-to-bedside review: Latest results in hemorrhagic shock. Crit Care 12: 218, 2008.

4. Cai B, Deitch EA and Ulloa L: Novel insights for systemic inflammation in sepsis and hemorrhage. Mediators Inflamm 2010: 642462, 2010.

5. Ananthakrishnan P, Cohen DB, Xu DZ, Lu Q, Feketeova E and Deitch EA: Sex hormones modulate distant organ injury in both a trauma/hemorrhagic shock model and a burn model. Surgery 137: 56-65, 2005.

6. Cheifetz IM: Year in review 2015: Pediatric ARDS. Respir Care 61: 980-985, 2016.

7. Hoegl S, Brodsky KS, Blackburn MR, Karmouty-Quintana H, Zwissler B and Eltzschig HK: Alveolar epithelial A2B adenosine receptors in pulmonary protection during acute lung injury. J Immunol 195: 1815-1824, 2015.

8. De Luca D, Piastra M, Tosi F, Pulitanò S, Mancino A, Genovese O, Pietrini D and Conti G: Pharmacological therapies for pediatric and neonatal ALI/ARDS: An evidence-based review. Curr Drug Targets 13: 906-916, 2012.

9. Lee CC, Chang IJ, Yen ZS, Hsu CY, Chen SY, Su CP, Chiang WC, Chen SC and Chen WJ: Delayed fluid resuscitation in hemorrhagic shock induces proinflammatory cytokine response. Ann Emerg Med 49: 37-44, 2007.

10. Claridge JA, Schulman AM and Young JS: Improved resuscitation minimizes respiratory dysfunction and blunts interleukin6 and nuclear factor-kappa B activation after traumatic hemorrhage. Crit Care Med 30: 1815-1819, 2002.

11. Jiang H, Huang Y, Xu H, Hu R and Li QF: Inhibition of hypoxia inducible factor- $1 \alpha$ ameliorates lung injury induced by trauma and hemorrhagic shock in rats. Acta Pharmacol Sin 33: 635-643, 2012.

12. Koscsó B, Trepakov A, Csóka B, Németh ZH, Pacher P, Eltzschig HK and Haskó G: Stimulation of A2B adenosine receptors protects against trauma-hemorrhagic shock-induced lung injury. Purinergic Signal 9: 427-432, 2013.

13. Hammond SM: An overview of microRNAs. Adv Drug Deliv Rev 87: 3-14, 2015

14. Soifer HS, Rossi JJ and Saetrom P: MicroRNAs in disease and potential therapeutic applications. Mol Ther 15: 2070-2079, 2017.

15. Krol J, Loedige I and Filipowicz W: The widespread regulation of microRNA biogenesis, function and decay. Nat Rev Genet 11: 597-610, 2010.

16. O'Connell RM, Rao DS, Chaudhuri AA and Baltimore D: Physiological and pathological roles for microRNAs in the immune system. Nat Rev Immunol 10: 111-122, 2010.

17. Tutar Y: miRNA and cancer; computational and experimental approaches. Curr Pharm Biotechnol 15: 429, 2014.

18. Vickers KC, Rye KA and Tabet F: MicroRNAs in the onset and development of cardiovascular disease. Clin Sci (Lond) 126: 183-194, 2014.

19. Zhang J, Liu Y and Lu L: Emerging role of microRNAs in peripheral nerve system. Life Sci 207: 227-233, 2018.

20. Karolina DS, Tavintharan S, Armugam A, Sepramaniam S, Pek SLT, Wong MTK, Lim SC, Sum CF and Jeyaseelan K: Circulating miRNA profiles in patients with metabolic syndrome. J Clin Endocrinol Metab 97: E2271-E2276, 2012.

21. Wang W, Tang S, Li H, Liu R, Su Y, Shen L, Sun M and Ning B: MicroRNA-21a-5p promotes fibrosis in spinal fibroblasts after mechanical trauma. Exp Cell Res 370: 24-30, 2018.

22. Ferruelo A, Peñuelas Ó and Lorente JA: MicroRNAs as biomarkers of acute lung injury. Ann Transl Med 6: 34, 2018.

23. Wang ZM, Wan XH, Sang GY, Zhao JD, Zhu QY and Wang DM: miR-15a-5p suppresses endometrial cancer cell growth via Wnt/ $\beta$-catenin signaling pathway by inhibiting WNT3A. Eur Rev Med Pharmacol Sci 21: 4810-4818, 2017. 
24. Chen D, Wu D, Shao K, Ye B,Huang J and Gao Y: MiR-15a-5p negatively regulates cell survival and metastasis by targeting CXCL10 in chronic myeloid leukemia. Am J Transl Res 9: 4308-4316, 2017.

25. Long J, Jiang C, Liu B, Fang S and Kuang M: MicroRNA-15a-5p suppresses cancer proliferation and division in human hepatocellular carcinoma by targeting BDNF. Tumour Biol 37: 5821-5828, 2016.

26. Kontos CK, Tsiakanikas P, Avgeris M, Papadopoulos IN and Scorilas A: miR-15a-5p, a novel prognostic biomarker, predicting recurrent colorectal adenocarcinoma. Mol Diagn Ther 21 453-464, 2017.

27. Verstrepen L, Carpentier I, Verhelst K and Beyaert R: ABINs: A20 binding inhibitors of NF-kappa B and apoptosis signaling. Biochem Pharmacol 78: 105-114, 2009.

28. Xie H, Yang M, Zhang B, Liu M and Han S: Protective role of TNIP2 in myocardial injury induced by acute pancreatitis and its mechanism. Med Sci Monit 23: 5650-5656, 2017.

29. Wang W, Gao J and Wang F: MiR-663a/MiR-423-5p are involved in the pathogenesis of lupus nephritis via modulating the activation of NF- $\mathrm{KB}$ by targeting TNIP2. Am J Transl Res 9: 3796-3803, 2017.

30. Guo S, Jiang K, Wu H, Yang C, Yang Y, Yang J, Zhao G and Deng G: Magnoflorine ameliorates lipopolysaccharide-induced acute lung injury via suppressing NF- $\mathrm{BB}$ and MAPK activation Front Pharmacol 9: 982, 2018.

31. Yu J, Ni L, Zhang X, Zhang J, Abdel-Razek O and Wang G: Surfactant protein D dampens lung injury by suppressing NLRP3 inflammasome activation and NF- $\mathrm{KB}$ signaling in acute pancreatitis. Shock 51: 557-568, 2018.

32. Yang G, Peng X, Hu Y, Lan D, Wu Y, Li T and Liu L: 4-phenylbutyrate benefits trau matic hemorrhagic shock in rats by attenuating oxidative stress, not by attenuating endoplasmic reticulum stress. Crit Care Med 44: e477-e491, 2016.
33. Shi HP, Deitch EA, Da Xu Z, Lu Q and Hauser CJ: Hypertonic saline improves intestinal mucosa barrier function and lung injury after trauma-hemorrhagic shock. Shock 17: 496-501, 2002 .

34. Liu WJ, Zhong ZJ, Cao LH, Li HT, Zhang TH and Lin WQ: Paclitaxel-induced lung injury and its amelioration by parecoxib sodium. Sci Rep 5: 12977, 2015.

35. Livak KJ and Schmittgen TD: Analysis of relative gene expression data using real-time quantitative PCR and the 2(-Delta Delta C(T)) method. Methods 25: 402-408, 2001.

36. Senthil M, Watkins A, Barlos D, Xu DZ, Lu Q, Abungu B, Caputo F, Feinman R and Deitch EA: Intravenous injection of trauma-hemorrhagic shock mesenteric lymph causes lung injury that is dependent upon activation of the inducible nitric oxide synthase pathway. Ann Surg 246: 822-830, 2007.

37. Vallabhapurapu S and Karin M: Regulation and function of $\mathrm{NF}-\kappa \mathrm{B}$ transcription factors in the immune system. Annu Rev Immunol 27: 693-733, 2009.

38. Mariappan N, Elks CM, Sriramula S, Guggilam A, Liu Z, Borkhsenious $\mathrm{O}$ and Francis J: NF-kappaB-induced oxidative stress contributes to mitochondrial and cardiac dysfunction in type II diabetes. Cardiovasc Res 85: 473-483, 2010.

39. Pateras I, Giaginis C, Tsigris C, Patsouris E and Theocharis S: $\mathrm{NF}-\kappa \mathrm{B}$ signaling at the crossroads of inflammation and atherogenesis: Searching for new therapeutic links. Expert Opin Ther Targets 18: 1089-1101, 2014.

This work is licensed under a Creative Commons Attribution-NonCommercial-NoDerivatives 4.0 International (CC BY-NC-ND 4.0) License. 